\title{
Cytoskeleton and CRMPs in Neuronal Morphogenesis and Neurological Diseases: Potential Targets for New Therapies
}

\author{
Quach $\mathrm{TT}^{1,2}$, Auvergnon $\mathbf{N}^{1}$, Lerch $\mathrm{JK}^{2}$, Honnorat $\mathrm{J}^{1,3,4}$, Khanna $\mathbf{R}^{5}$, Kolattukudy $\mathrm{PE}^{6}$ and Duchemin $\mathrm{AM}^{7^{*}}$ \\ ${ }^{1}$ Lyon Neuroscience Research Center INSERM U1028/CNRS UMR 5292, F-69372 Lyon, France \\ ${ }^{2}$ Department of Neuroscience, Center for Brain and Spinal Cord Repair, The Ohio State University, Columbus, OH, 43210, USA \\ ${ }^{3}$ French Reference Center on Paraneoplastic Neurological Syndrome, Hospices Civils de Lyon, Hôpital Neurologique, Neurologie B, F-69677 Bron, France \\ ${ }^{4}$ Université de Lyon - Université Claude Bernard Lyon 1, F-69372 Lyon, France \\ ${ }^{5}$ Department of Pharmacology, College of Medicine, The University of Arizona, Tucson, AZ, 85724. USA \\ ${ }^{6}$ Burnett School of Biomedical Sciences, College of Medicine, University of Central Florida, Orlando, FL, 32816, USA \\ ${ }^{7}$ Department of Psychiatry, College of Medicine, The Ohio State University. Columbus, OH, 43210, USA \\ "Corresponding author: Duchemin AM, Department of Psychiatry, College of Medicine, The Ohio State University, Columbus, OH, 43210, USA, Tel: (614) 293-4540; E- \\ mail: duchemin.1@osu.edu
}

Received date: January 01, 2016; Accepted date: January 22, 2016; Published date: January 25, 2016

Copyright: (c) 2016 Quach, et al. This is an open-access article distributed under the terms of the Creative Commons Attribution License, which permits unrestricted use, distribution, and reproduction in any medium, provided the original author and source are credited.

\begin{abstract}
Cytoskeletal proteins, and the molecules that control their assembly/disassembly, regulate neurite and spine growth and retraction, which are necessary for normal brain function. The neuron begins as a spherical shape, then lamellipodia and filopodia form nascent neurites which differentiate into dendrites and axons. These processes, also involved in neuronal plasticity, require changes in the dynamics of the cytoskeleton proteins and their intracellular binding partners, including collapsin-response mediator proteins (CRMPs). Abnormal changes in CRMP signaling induce structural/ functional abnormalities in neurons which are characteristics of various neurological disorders. Modulation of these pathways may represent unexplored areas for treatment of these diseases.
\end{abstract}

Keywords: CRMP; Cytoskeleton; Neuritogenesis

\section{Introduction}

Neurons integrate and transmit information to allow a living animal to dynamically adapt to environmental changes over time from development to aging. Through the process of neurite specification, arborization, branching, outgrowth, and pruning of inappropriate dendritic and/or axonal branches, an individual neuron interacts efficiently with multiple targets. The neurite development and arborization process requires specific changes in the dynamics of the cytoskeleton, particularly tubulin and actin, and their intracellular binding partners including CRMPs. Regulatory mechanisms maintain the neurite arborization structure by balancing plasticity versus stability. Accordingly, direct changes in the intracellular signaling pathways that affect cytoskeletal dynamics or changes secondary to the increase or decrease of neurotrophic factors and permissive/inhibitory guidance cues may induce structural abnormalities in neurons. These abnormalities can alter information processing, and neuronal network formation/maintenance and connections, which are characteristic of many neurological disorders. CRMPs bind to cytoskeletal proteins and regulate neurite growth, maintenance and plasticity. CRMP activity is regulated at the transcription level by splice variants lacking specific binding sites, and post-translationnally by phosphorylation by various kinases that affects their binding affinity to their effectors. Interestingly, many neurological disorders with abnormalities in cytoskeletal structure/organization are associated with CRMPs dysfunction. The primary intent of this review is to define the functional roles of CRMPs and their cytoskeletal binding partners in neurite formation and maintenance as they relate to their possible involvement in several neurological disorders characterized by structural abnormalities of neurites. Recent investigations on the modulation of cytoskeletal proteins and CRMPs' activity suggest new potential targets for therapeutic intervention in these diseases.

\section{Cytoskeleton and CRMPs Dynamics in Neuronal Morphogenesis}

Although neurons must be able to change their shape during different phases of development to achieve specific functions, in adults, despite the great diversity of morphology between neuronal types, most vertebrate nerve cells exhibit distinctive dendrite morphology and specific dendritic/axonal arbor/ramification depending on their location. The axono-dendritic arbor which influences the number of synaptic inputs that each neuron can integrate emerges as a convergent product of specific pattern of growth, branching and retraction and is differentially regulated at multiple points, including the control of the number of primary branches and their mode and frequency of branching. The cellular cytoskeleton and molecules regulating its dynamics mediate these changes [1,2]. Accordingly, disruption of the activity of these proteins may lead to or be associated with various neurological disorders.

\section{Lamellipodia formation, neuritogenesis and dendrite morphogenesis}

Cytoskeleton:Establishment of lamellipodia is considered a principal means of initiating primary neurites formation. Lamellipodia are thin protrusions of membrane roughly $140 \mathrm{~nm}$ long surrounding the cell. They contain a stretched meshwork and bundles of branched F-actin 
filaments having their barbed ends directed towards the cell membrane. F-actin assembles from ATP-bound G-actin monomers and requires many actin-regulating proteins such as cofilin, ADF, MARCKS, PIP2, N-WASP, MAPs, Arp2/3, Ena/Vasp, profilin and filamin [3-6]. Over time, lamellipodia undergo segmentation. The segmented areas in which actin subunit addition/filament elongation occurs gradually extend away from the leading edge to allow these structures to form filopodia, critical elements for neurite formation. The specific repertoires of proteins that orchestrate this complex sequence of morphological events, including their engagement in a spatio-temporal manner to organize different actin isoforms, microtubule arrays -and their reciprocal interaction through MAPsand the molecular mechanisms of the protrusion of the leading edge membrane remain largely unknown. Furthermore, recent studies provide evidence that there are multiple signaling pathways/molecular mechanisms that can initiate filopodia formation or its retraction even in the same cell [7]. Despite the variability of dendrite arbors, dendrites share some fundamental characteristics: there is abundant evidence that their development is determined by various intrinsic/extrinsic codes. During development, neurons likely encounter similar environment factors. Intrinsic pathways (Ras, RhoA, Rac, Cdc42, Tiam-1, CaMKII, GSK3 $\beta$, mTOR) within each neuron control the cellular interpretation of the extrinsic cues to allow neurons to generate different patterns of dendrite development [8-10]. In addition, various intrinsic transcription factors (Foxo6, Neurogenin, NeuroD, CREB, CREST, Sp4, MEP2A) may also operate with different extrinsic chemo-attractive and chemo-repulsive cues such as semaphorins, ephrin, Slit, reelin, VGCC, neurotrophins, extracellular matrix, to facilitate the tiling/self-avoidance mechanisms (Dscam; Stop Signal Notch1), to tailor the dendritic shape in response to the new environment or various stimuli, and importantly to maintain an equilibrium between dendrite dynamics and stabilization for each distinct dendrite arbor morphology [11,12]. Ultimately, all these processes are intimately tied to their effect on the proper dynamic of cytoskeletal proteins through the Rho family of GTPases.

CRMPs: CRMPs represent a family of proteins that binds to cytoskeleton proteins and are involved in the regulation of dendrite and axon growth and retraction. In addition to neuron cell cultures, gene-targeted inactivation in rodents has enabled elucidation of unique functions of individual CRMPs in different cell types in vivo. In culture, neurons from Crmp3-/- knockout mice cannot break the cellular symmetry to extend neurites and establish neuronal polarity, demonstrating that CRMP3 is essential in the early stage of neurite initiation and dendrite formation [13]. Consistent with this idea, CRMP3 over-expression promotes important lamellipodia formation and significantly increases dendritic number, length and branching per neuron [14]. In contrast, CRMP5 depletion by RNAi enhances the length and number of neurites while its over-expression induces mitophagy and reduces dendrite length without affecting primary dendrite formation. Further, over-expression of a truncated CRMP5 isoform devoid of the tubulin-binding domain has no effect on dendrite length or formation, demonstrating the critical role of tubulin-binding domain in the neurite inhibitory outgrowth of CRMP5 [15]. CRMP2 has been reported to enhance dendrite lengths. CRMP5 and CRMP2 both interact with the tubulin complex and study on the combinatorial functions of both CRMP2-CRMP5 led to the finding that the neurite outgrowth-promoting function of CRMP2 is totally abolished by CRMP5, which acts as a dominant negative signal [15]. Increasing evidence supports CRMP4 involvement in regulation of dendritic outgrowth. In Crmp4-/- mice, the distance from the soma to the first bifurcation point in the apical dendrites of CA1 pyramidal neurons is significantly decreased, confirming an inhibitory role of CRMP4 on dendrite bifurcation and branching [16]. CRMP1 is mainly localized in dendrites of various brain structures and its temporal light-mediated inactivation promotes retraction of lamellipodia followed by retardation of neurite formation. In addition, Crmp1-/mice display abnormal GAP43/PSD95/MAP2/Golgi staining in the CA1 area of hippocampus, consistent with structural dendritic alteration $[17,18]$.

\section{Axonogenesis}

Cytoskeleton:During brain development, neurons display a dazzling diversity of distinct axonal phenotypes identified by their complexity of axonal arbor structures with distinct branching management (basket cell axons vs parallel fibers of cerebellum) and diameters (myelinated vs unmyelinated fibers). Axon elongation is ultimately achieved through microtubule assembly and extension, actin reorganization and membrane genesis at the leading edge of growth cones. It has been generally assumed that the axon-dendrite polarity involves polarized transport of various cargoes, complex arrays of microtubule orientation -axons contain microtubules oriented uniformly plus-end distal while dendrites have microtubules in a mixed orientation through dynein function-, and distinct repertoires of ECM/structural/ signaling proteins. For example, 1) the multipolar cells might receive extrinsic factors (TAG-1) from the surrounding microenvironment to establish polarity and differentiate into bipolar cells, 2) down regulation of MAP2 inhibits dendrite formation whereas down regulation of Taul inhibits axon formation. After the neuron polarizes, the axon may extend a long distance to reach its targets and form presynaptic structures and the kinase GSK-3 $\beta$ is the main intracytoplasmic-integrating signal that translates the extracellular codes into controlled axonal extension and guidance through various substrates, including CRMP2, APC, Tau, Stathmin, MAP1b, Katanin, KIF2C, myosin2 and CLAPS1/2 [19-21]. Another significant difference in axonal phenotype is the proximal axon characterized by the axonal initial segment (AIS) which is a microtubule-based filter that retains Tau in the axon while Spectrin and Ankyrin B form the basis of the plasma membrane barrier [22].

CRMPs: It has been generally assumed that specification in axon or dendrite involves specific proteins. However, several molecules such as CRMP2 can affect both dendrites (as discussed above) and axons. Overexpression of CRMP2 in hippocampal neurons induces supernumerary axons, axon elongation, and increases axonal branching while its inhibition with a dominant negative protein suppresses the formation of primary axon [23]. CRMP2 can also convert existing dendrites into axon. Mechanisms for the regulation of the axonal growth may involve changes in microtubule polymerization, participation in the Sra-1/WAVE/kinesin cargo complex and modulation of vesicle/membrane trafficking/remodeling through interaction with Numb, depending upon specific post-translational modifications $[24,25]$. CRMP2 is an effector of secreted neurotrophic and neurite collapsing factors. BDNF and NT3, by inhibiting CRMP2 phosphorylation by GSK-3 $\beta$, induce axonal growth and branching activity while Sema-3A, by increasing CRMP2 phosphorylation via GSK-3 $\beta$, induces axonal retraction [26]. Ectopic expression of the short-isoform of CRMP4 also enhances axonal extension whereas it dephosphorylated, long-isoform mediates MAI-induced neurite outgrowth inhibition via actin-dependent processes [27]. These observations suggest that CRMP2 phosphorylation levels and CRMP4 
isoform ratio represent possible mechanisms of regulation of axonal structure/ function.

Immunocytochemical and mutant protein studies of CRMP1 detected a reduction in axonal extension induced by NT3 in neurons from dorsal root ganglia [28]. However, axonal length from cerebellar explants cultures of Crmp1-/- mice are similar to wild type [18]. In contrast to their role in dendritogenesis, CRMP3 and CRMP5 deletion or overexpression yields no apparent effect on axon morphogenesis in cultured hippocampal CA1 neurons and cultured cerebellar explants. Considering the multifaceted phenotypes of CRMPs-deficient mice from different contexts, it is not surprising that these proteins are found altered in several neurological disorders [2].

\section{Neurological Diseases: A Focus on CRMPs and Cytoskeleton}

\section{Alzheimer's disease (AD)}

The cellular hallmark of $\mathrm{AD}$ is dendritic dystrophy, including reduction of dendrite length/complexity, and fragmentation and alteration of spine density/shape. The molecular hallmark is the defect and collapse in cytoskeletal proteins which represents the seminal event described as senile plaques, neurofibrillary tangles (NFTs) and cofilin pathology: Tau in promoting tubulin polymerization plays a critical role in tubulin dynamic. Tau's aberrant hyperphosphorylation by GSK-3 $\beta / \mathrm{Cdk} 5$ alters its ability to bind microtubules resulting in their disassembly; actin and cofilin may also be involved [29]. Alzheimer patients present a decline in cognitive ability beginning with the loss of short term memory. The hippocampus -with prominently reduced dendritic complexity- is one of the earliest and most vulnerable brain regions affected by the disease [30]. Interestingly, increased phosphorylated CRMP2 has been detected in the soluble fraction of $\mathrm{AD}$-affected brain tissue but not in other types of dementia [31]. The hippocampal synaptic dysfunction and memory impairments of $\mathrm{AD}$ are modeled in $\mathrm{AD}$ transgenic mice with neuritic senile plaques ( $\beta$-amyloid) and NFTs, supporting the neuropathological concept of a 'dendritic-dysconnectivity' syndrome [32]. In transgenic AD mouse models, phosphorylated CRMP2 is increased in hippocampus well before plaque and tangle formation suggesting that it is an early event in the pathogenesis of the disease [33]. Although the relationship between pathophysiological mechanisms and $\mathrm{AD}$ symptoms remains to be clarified, these findings position phosphorylated CRMP2 as a potential biomarker for AD early detection. Of note, in the 6-hydroxydopamine (6-OHDA) model of Parkinson disease, which is associated with alteration in microtubule integrity, CRMP2 is up-regulated in brain regions including the corpus callosum, an area where CRMP2 is normally not present [34].

\section{Prion diseases}

In the sporadic/familial Creutzfeld-Jacob disease and other prion diseases, cellular alpha-helix rich prion protein $\left(\mathrm{PrP}^{\mathrm{c}}\right)$ is converted to the beta-structure-rich insoluble abnormal protease-resistant conformer $\left(\mathrm{PrP}^{\mathrm{Sc}}\right)$ [35]. These diseases are characterized by dendritic dystrophy followed by neuronal loss, gliosis and spongiform changes in the brain leading to dementia/ataxia. In vitro experimental procedures have shown a direct interaction between $\operatorname{PrP}$ and $\alpha / \beta$-isoform of tubulin. $\operatorname{PrP}$ has been reported to disassemble the microtubules cytoskeleton (Figure 1C and 1B) leading to a loss of neurites and necrotic cell death. A specific increase in CRMP4 expression and dendritic degeneration are the two earliest morphological features detectable in mouse models of prion disease before clinical symptoms are evident $[36,37]$. In the late stage of the disease, the amount of a truncated CRMP2 (CRMP2- $\Delta \mathrm{C}$ ), which lacks the sequence from Ser-518 to the C-terminus) is increased significantly [38]. Consistent with these evidences, we observed that the cytotoxic peptide $\operatorname{PrP}^{106-126}$ induces dendrite dystrophy in cultured neurons as well as an alteration of CRMP2 expression and a profound disturbance of CRMP2 electrophoretic pattern (Figure 1A). This effect was prevented by inhibition of calpain, suggesting that a calpain-mediated alteration of CRMP2 may result in disruption of microtubules, leading to pathogenic effects (Figure 1A and 1D).

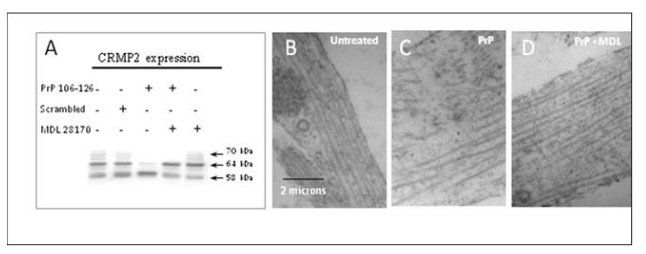

Figure 1: Changes in CRMP2 and microtubules after treatment with prion peptide: Association of CRMP2 calpain-mediated cleavage with cytoskeletal perturbation in PrP106-126 treated neurons. The balance between the different forms of CRMP2 was drastically changed in neurons treated with PrP106-126 (A lane 3) but not in neurons treated with scrambled peptide (A lane 2), the upper bands being reduced concomitantly with a marked increase of the $58 \mathrm{kDa}$ product analyzed by western blot. Transmission electron micrograph studies show that the change in CRMP2 induced by PrP106-126 is associated with microtubule network disorganization (C) compared to control (B). Inhibition of calpain by MDL 28170 not only prevents the change in CRMP2 (A lane 3) but also rescues neurons from microtubule breakdown (D). Thus in prion disease, a calpain-mediated alteration of CRMP2 may result in disruption of microtubules, leading to pathogenic effects.

\section{Amyotrophic lateral sclerosis (ALS)}

ALS is a multifaceted neurodegenerative disease influenced by varied genetic (mutation of SOD1; single nucleotide polymorphism (SNPs) of C9ORF72)/epigenetic (neurotoxin from cyanobacteria; pesticides) factors affecting upper and lower motor neurons [39]. Other cellular and molecular experiments have identified defects in growth factors, axonal transport or defect in RNA processing. Data from clinical and animal studies support the idea that ALS is a distal "dying-back" axonopathy that occurs at very early stage at the neuromuscular synapses prior to symptom onset, with important disturbance of microtubules and actin structure and impaired axonal transport [40,41]. In this scenario and as CRMP2 links the cytoskeleton microtubule to the molecular motors, it is tempting to suggest that CRMP2 activity may also be part of the development of the presymptomatic stages of ALS. Moreover, the stable tubule only polypeptide (STOP), various guidance cues including the repulsive axon Sema3A and its downstream effectors CRMPs, are linked to the pathophysiology of ALS [42]. It has been shown in animal models that expression of the long variant of CRMP4 is increased in a subpopulation of lumbar motor neurons in SOD1 mice starting at presymptomatic stages, and its over-expression in cultured 
Page 4 of 8

motoneurons leads to inhibition of neurite outgrowth followed by cell death.

\section{Epilepsy}

Next generation sequencing has led to the identification of an array of epilepsy genes [43]. Various animal and human tissue studies suggest that epilepsy development involves reorganization and sprouting of hippocampal mossy fibers, and changes in cytoskeleton dynamics, calcium signaling and CRMP expression [44-46]. The nexus between CRMP2 and epilepsy was recently highlighted by recent studies on posttraumatic epilepsy, the development of temporal lobe epilepsy (TLE) following traumatic brain injury, which accounts for $20 \%$ of symptomatic epilepsies. Reorganization of mossy fibers within the hippocampus is a common pathological finding of TLE and likely in combination with other mitigating factors contributes to epileptogenesis. During TLE, mossy fibers innervate the inner molecular layer of the hippocampus where they synapse onto the dendrites of other dentate granule cells, leading to the formation of recurrent excitatory circuits. Although the exact role of CRMP2 in mossy fiber sprouting has not yet been determined and a causal relationship has yet to be drawn between mossy fiber sprouting and epileptogenesis, it is possible that the loss of GSK- $3 \beta$ phosphorylation immediately following injury contributes to the induction of mossy fiber sprouting while the loss of priming by CDK5 in later phases contributes to its maintenance. It is of great interest that these mechanistically distinct events culminate in a similar end-point: an increase in the amount of active CRMP2 (Figure 2). At the very least, mossy fiber sprouting is linked to the exacerbation of the progression of the disease as well as the manifestation of its symptoms. The involvement of CRMP2 in such processes, however, remains untested.

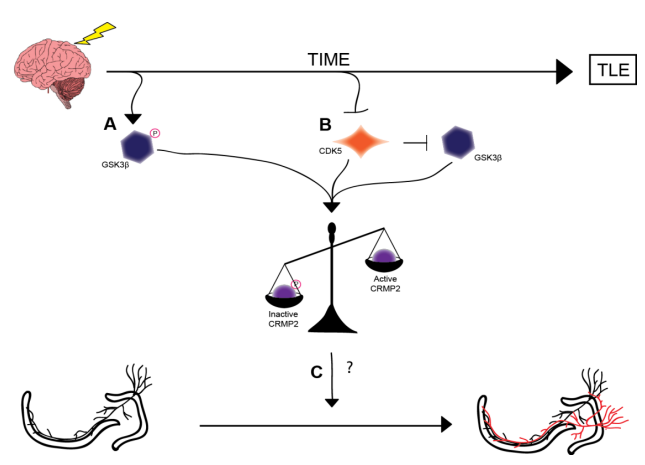

Figure 2: Graphical summary of TLE model: (A) GSK-3 $\beta$ is phosphorylated and thereby inactivated in the early phases following injury. This inactivation leads to decreased amounts of phosphorylated (inactive) CRMP2. (B) CDK5 phosphorylation of CRMP2 is decreased in the later phases following injury. This decrease in phosphorylation also indirectly reduces levels of GSK-3 $\beta$-phosphorylated CRMP2 through a loss of priming, resulting in an overall increase in the proportion of active CRMP2. (C) The sustained increase in unphosphorylated (active) CRMP2 in the hippocampus may underlie aberrant mossy fiber sprouting following injury.

\section{Paraneoplastic neurological disorders (PND)}

The neuronal damage of PNDs is caused through a remote immuneeffect of the tumor on the nervous system. Auto-antibodies against CRMP5 (also called anti-CV2 or anti-CV2/CRMP5) have been described in a subgroup of PND [47]. Patients with these antibodies frequently show peripheral sensorimotor neuropathy, cerebellar ataxia, limbic encephalitis, and chorea. The identification of CRMP5 as the main target of anti-CV2 antibodies in PND patients is important for understanding the pathophysiology of the disease [48]. Indeed, Crmp5-/- mice mimic PND neurologic pathology and symptoms resulting from damage of the peripheral and central neurons, and exhibit peripheral neuropathy with abnormal motor conduction velocities, abnormal polyaxonal ensheathment, abnormal Schwann cell structure, as well as altered Purkinje dendrites in cerebellum with abnormal limb-clasping reflex and abnormal long term depression, suggesting that the various symptoms in PND patients can be linked to the CRMP5 protein [49].

\section{Autism spectrum disorders (ASD)}

A consistent feature of neurons in ASD patients is abnormal dendritic structure with alterations in dendritic spine morphology. The discovery of single gene mutations of several genes involved in cytoskeletal proteins ( $M E C P 2$ involved in the regulation of neuronal $\alpha$ tubulin expression, $S H A N K 3$ which has a pivotal role in the formation and maturation of spine via an actin-dependent mechanism, the neurexin gene family- and cytoskeleton dynamics -LIMK1,PAK3, $A R F G E F 6$, 9), copy number variations, chromosome abnormalities, changes in protein expression (altered neuritogenesis mediated by altered cellular $\mathrm{PrP}^{\mathrm{c}}$ levels), and epidemiological twin studies suggest that genetic abnormalities play important role in ASD pathogenesis [50-52]. Of note, it has been found that AUTS2 (the Autism susceptibility candidate 2 gene) induces lamellipodia but suppress filopodia formation. On the other hand, the findings of the presence of maternal autoantibodies directed against fetal brain tissues together with the passively transferred human IgG from mother brain-reactive antibodies studies provide strong evidence that maternally derived antibodies could lead to neurodevelopmental alterations in a subgroup of ASD. In this context, it has been shown that maternal autoantibodies reactive to cypin, CRMP1 and CRMP2 confer an increased risk for autism [53].

\section{Neuropathic pain (NP)}

Associated with damage/trauma to central or peripheral nervous system caused by a primary injury or by numerous diseases, NP may be related to abnormal sprouting and spine remodeling that lead to neuron hyperexcitability and decreased in pain threshold. Three points are important to emphasize. First, proteomic studies identify that changes in cytoskeletal tubulin3, calcium channels, CRMP2 and MAP2 are associated with NP in animal models. Second, CRMP5 expression is altered in injured and regenerating stages of sciatic nerve. Third, $\mathrm{N}$ type VGCC (CaV2.2) are genetically and clinically validated targets for pain management, and uncoupling these proteins from CRMP2, with which they bind, is anti-nociceptive in various models of neuropathic pain [54-56]. Thus, the indirect change of CaV2.2 by regulating its interaction with CRMP2 may afford better safety and efficacy profile than currently available CaV2.2-selective blockers which have many untoward side effects [57]. 


\section{Therapeutic Perspectives}

It is clear that the cytoskeleton and CRMPs play a critical role in maintaining neuronal phenotype and activity and their alteration affect the axono-dendritic arbor -which is a major site of histopathologic alterations in many neurological diseases. Alterations in synaptic structure with increased connectivity may impact epilepsy, neuropathic pain, and autism while decreased function may be related to neurodegenerative disorders such as $\mathrm{AD}$ and prion disease or lead to muscle atrophy and fasciculation as in ALS. Few available treatments in humans are regulating CRMPs and cytoskeletal proteins, and targeting them specifically is explored in various animal models.

\section{Actin as a potential biological therapeutic target}

Cumulative clinical and histopathological evidence suggests that memory loss correlates better with synapse/spine loss than with plaques or tangles formation in AD. Genetic studies further demonstrate a significant causal link between spine density/structure and the cognitive deficit via a change in drebrin - a dendritic spine actin-regulating protein, and fractin - a caspase-cleaved fragment of actin, and the fractin/actin ratio. That actin is a possible therapeutic target can be surmised from the following observations: 1) docosahexaenoic acid (DHA), an essential fatty acid which prevents caspase cleavage of actin, decreases the likelihood of developing $\mathrm{AD}$ and 2) urokinase-type plasminogen activator (uPA), which shifts the actin pool from G-actin to F-actin, induces the re-emergence of filopodia and spines during the recovery phase of brain injury $[58,59]$.

\section{Microtubule as potential biological therapeutic target}

Alteration in microtubule spatio-temporal regulation by their molecular binding partners (MBP) has negative consequence on axonal transport and neuronal function, which recently lead to proposing microtubules/MBPs as promising therapeutic targets for treatment of nervous system disorders and injury [60,61]. Accordingly, recent studies demonstrate that microtubule-stabilizing agents used as anticancer drugs can yield beneficial outcomes in promoting axonal regeneration and ameliorating the pathogenic symptoms in mice models of neurodegenerative disorders: low doses of paclitaxol promote serotonergic axon regeneration, reduce macrophage infiltration and decrease scar formation at spinal cord injury sites while epothilone improves cognition of tau-transgenic mice models $[62,63]$. These findings indicate that microtubule and MBPs may offer effective targets to control multiple pathways to produce better outcome in brain disorders treatment [64].

\section{CRMPs as potential biological therapeutic targets}

Under various physiological and pathological conditions during neuron development, CRMPs are critical signal transducers and intracytoplasmic regulators involved in structuring cytoskeletal proteins activity and dynamic. Accordingly, CRMPs hold considerable promise as therapeutic agents. Indeed, a 15 amino acid CRMP-2derived peptide (designated as tat-CBD3) that disrupts the CaV2.2/ CRMP2 interaction has proved to be anti-nociceptive in preclinical animal models of inflammation and neuropathic pain [54-57,65]. $\mathrm{AAV}$-encoded $\mathrm{CBD} 3$ delivered to rat peripheral sensory neurons through DRG injection provides sustained relief ( $>6$ weeks) of NP [66]. The positive effect and relative lack of toxicity of the AAVtargeted expression of CBD3 peptide in this animal model suggest that CBD3 may be a valuable approach, not only for exploring the role of presynaptic VGCCs and long-term modulation of neurotransmission, but to treat chronic NP and other disorders [67].

In neurons in culture, it was found that PrP106-126 induced dystrophic changes in dendrites, an effect prevented by Taxol treatment that interferes with the normal breakdown of microtubules (Figure 3) and by overexpression of CRMP3 [14]. In patients with prion pathology, the extensive neuronal loss associated with the spongiform degeneration affects the basal ganglia and the cerebral and cerebellar cortex whereas the CRMP3-rich hippocampal formation is protected until the final stage of disease $[68,69]$. The high levels of CRMP3 expression in hippocampus may explain, at least in part, the relative protection of this structure from the disease, and suggests that CRMP3 may prove useful in the prevention of altered dendritic structure in vivo. Similarly, the fact that CRMP2 expression and/or phosphorylation/activity are altered in Alzheimer's disease raises the attractive hypothesis that the protein may be a viable therapeutic target [70]. In an animal model of $\mathrm{AD}$, suppression of CRMP2 phosphorylation is associated with amelioration of $\beta$-amyloid-induced cognitive dysfunction and hippocampal axon degeneration [70].
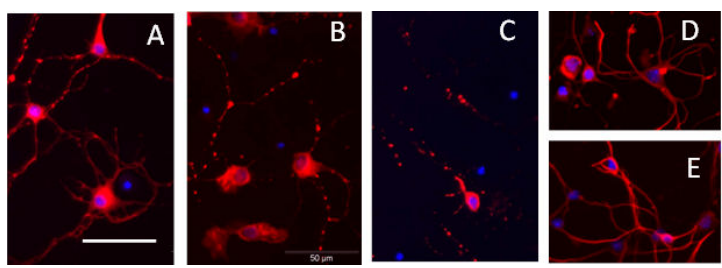

Figure 3: Effects of microtubule stabilizing agents on cells treated with prion peptide: Primary neurons ( $\beta$-tubulin positive) subjected to different treatments: untreated cells (A), PrP106-126 $80 \mu \mathrm{M}$ for 2 h (B), PrP106-126 $80 \mu \mathrm{M}$ for $24 \mathrm{~h}$ (C), PrP106-126 $80 \mu \mathrm{M}$ and Taxol (a microtubule-stabilizing agent) $10 \mu \mathrm{M}$ for $24 \mathrm{~h}$ (D), or Taxol 10 $\mu \mathrm{M}$ for $24 \mathrm{~h}(\mathrm{E})$. All micrographs are represented with the same total magnification. White bar: 50 microns.

There is no efficient therapy for ALS patient, and new therapeutic targets need to be explored [71]. The observations of increased CRMP4 in animal models of ALS is further strengthened with adeno-associated virus (AAV)-mediated overexpression of CRMP4 in motoneurons in vivo that leads to significant muscle denervation and reduction in motoneuron numbers [72]. Altogether, these findings strongly suggest that CRMP4 may be an early effector candidate in the SOD1 mouse model of ALS and controlling its activity may ameliorate ALS symptoms.

Finally, S-lacosamide ((S)-LCM) -the enantiomer of Vimpat, an antiepileptic drug in clinical use- inhibits CRMP2 phosphorylation without off target effects but with functional consequence such as blocking calcium influx via inhibition of VGCCs [73]. The extent of mossy fiber sprouting, a hallmark of temporal lobe epilepsy (TLE) in rats that received (S)-LCM following a controlled cortical impact injury, is markedly decreased compared to controls [74]. This suggests that (S)-LCM, a small molecule, may be a novel tool with potential therapeutic applications not only for epilepsy but other neuropathologies (Figure 4). 


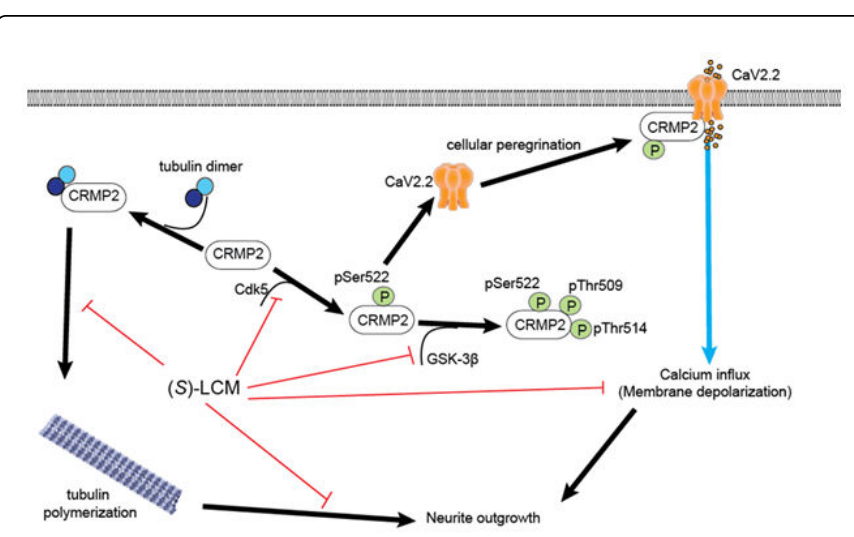

Figure 4: Working model summarizing the actions of (S)-LCM on CRMP2 phosphorylation and calcium signalling: CRMP2 is believed to contribute to neurite outgrowth by (i) binding to tubulin dimers, and (ii) via its GTPase-activating protein (GAP) activity, promoting tubulin polymerization. CRMP2 is phosphorylated at serine residue 522 by Cdk5, which 'primes' CRMP2 for subsequent phosphorylation by GSK-3 $\beta$. Cdk5-phosphorylated CRMP2 also has a greater propensity to interact in a complex with CaV2.2; the interaction also contributes to increased peregrination of the channel to the cell surface where it contributes to a greater amount of calcium influx. Influx via CaV2.2 is critical for changing the neuronal membrane potential culminating in enhanced neuronal activity. CRMP2 promotes neurite outgrowth in an activity-driven manner. (S)-LCM acts to (i) inhibit CRMP2-dependent tubulin polymerization which prevents growth of neurites, (ii) directly inhibits CRMP2 phosphorylation by Cdk5 and GSK-3 $\beta$, (iii) leading to a loss of binding to CaV2.2 and suppression of calcium influx via these channels, which collectively culminates in suppression of neurite outgrowth.

\section{Conclusion}

The CRMPs signaling system is a critical pathway that regulates neurite initiation, axono-dendritic growth/branching and retraction during neuronal maturation and plasticity and consequently affects neuronal connectivity/activity and behavioral responses. In agreement with this role, it is apparent that the cytoskeleton and CRMPs interactions may also be involved in neurological disorders associated with dendrite or axon dystrophy, atrophy or hypertrophy. Although presently the biochemical, pharmacological and electrophysiological data originate mostly from in vitro experimental models or from in vivo genetically modified mice and more rarely from patients samples, the proposed concept is compelling and worthy of continued research. Additional post-mortem-CRMPs-directed immunohistopathology observations, especially at different disease stages, and probing for specific CRMP splice variants and un/phoshorylated isoforms are needed to confirm the critical role of these proteins in brain diseases while SNP linkage, GWAS and meta-analyses of the polygenic score profile may reveal complexity in CRMP genetic contribution. Causality cannot be inferred from the human data but in vitro and in vivo animal model suggest possible mechanisms. Other molecules involved in cytoskeletal assembly are also likely to be associated with these diseases. However, the fact that CRMPs directly bind to tubulin to regulate assembly and disassembly of microtubules place them at the downstream end of the signaling pathways initiated by extracellular or intracellular factors controlling structural changes of neurites and spines. This can explain their association with various diseases and make them a perfect target for intervention in disorders associated with alterations of neurite structure. Since CRMPs activity is regulated by phosphorylation, kinase inhibitors represent a first way to investigate pharmacological modification of their activity. Small molecules increasing or decreasing the binding of CRMPs to their effectors represent another more specific strategy. Finally, unraveling the finer molecular details of how these different molecules interact under physiological and pathological conditions to regulate the CRMPs phosphorylation/cytoskeletal dynamics [75,76], associated with genomic studies may aid in the design of combinatorial therapies defying these devastating neurological diseases within the framework of personalized pharmacogenetical therapy.

\section{References}

1. Stiess M, Bradke F (2011) Neuronal polarization: the cytoskeleton leads the way. Dev Neurobiol 71: 430-444.

2. Quach TT, Honnorat J, Kolattukudy PE, Khanna R, Duchemin AM, (2015) CRMPs: critical molecules for neurite morphogenesis and neuropsychiatric diseases. Mol Psychiatry 20: 1037-1045.

3. Sainath R, Gallo G (2015) Cytoskeletal and signaling mechanisms of neurite formation. Cell Tissue Res 359: 267-278.

4. Svitkina TM, Bulanova EA, Chaga OY, Vignjevic DM, Kojima S, et al. (2003) Mechanism of filopodia initiation by reorganization of a dendritic network. J Cell Biol 160: 409-421.

5. Wagner AR, Luan Q, Liu SL, Nolen BJ (2013) Dip1 defines a class of Arp2/3 complex activators that function without preformed actin filaments. Curr Biol 23: 1990-1998.

6. Yamaguchi H, Shiraishi M, Fukami K, Tanabe A, Ikeda-Matsuo Y, et al. (2009) MARCKS regulates lamellipodia formation induced by IGF-I via association with PIP2 and beta-actin at membrane microdomains. J Cell Physiol $220: 748-55$.

7. Gallo G (2013) Mechanisms underlying the initiation and dynamics of neuronal filopodia: from neurite formation to synaptogenesis. Int Rev Cell Mol Biol 301: 95-156.

8. Scott EK, Luo L (2001) How do dendrites take their shape? Nat Neurosci 4: 359-365.

9. Gaudillière B, Konishi Y, de la Iglesia N, Yao G, Bonni A (2004) A CaMKII-NeuroD signaling pathway specifies dendritic morphogenesis. Neuron 41: 229-241.

10. Jaworski J, Sheng M (2006) The growing role of mTOR in neuronal development and plasticity. Mol Neurobiol 34: 205-219.

11. Santiago C, Bashaw GJ (2014) Transcription factors and effectors that regulate neuronal morphology. Development 141: 4667-4680.

12. Urbanska M, Blazejczyk M, Jaworski J (2008) Molecular basis of dendritic arborization. Acta Neurobiol Exp (Wars) 68: 264-288.

13. Quach TT, Massicotte G, Belin MF, Honnorat J, Glasper ER, et al. (2008) CRMP3 is required for hippocampal CA1 dendritic organization and plasticity. FASEB J 22: 401-409.

14. Quach TT, Wilson SM, Rogemond V, Chounlamountri N, Kolattukudy PE, et al. (2013) Mapping CRMP3 domains involved in dendrite morphogenesis and voltage-gated calcium channel regulation. J Cell Sci 126: 4262-73.

15. Brot S, Rogemond V, Perrot V, Chounlamountri N, Auger C, et al. (2010) CRMP5 interacts with tubulin to inhibit neurite outgrowth, thereby modulating the function of CRMP2. J. Neurosci 30: 10639-10654.

16. Niisato E, Nagai J, Yamashita N, Abe T, Kiyonari H, et al. (2012) CRMP4 suppresses apical dendrite bifurcation of CA1 pyramidal neurons in the mouse hippocampus. Dev Neurobiol 72: 1447-1457.

17. Su KY, Chien WL, Fu WM, Yu IS, Huang HP, et al. (2007) Mice deficient in collapsin response mediator protein-1 exhibit impaired long-term 
potentiation and impaired spatial learning and memory. J Neurosci 27 2513-2524.

18. Yamashita N, Takahashi A, Takao K, Yamamoto T, Kolattukudy P, et al. (2013) Mice lacking collapsin response mediator protein 1 manifest hyperactivity, impaired learning and memory, and impaired prepulse inhibition. Front Behav Neurosci 7: 216.

19. Hur EM, Zhou FQ (2010) GSK3 signalling in neural development. Nat Rev Neurosci 11: 539-551.

20. Yu W, Qiang L, Solowska JM, Karabay A, Korulu S, et al. (2008) The microtubule-severing proteins spastin and katanin participate differently in the formation of axonal branches. Mol Biol Cell 9: 1485-98.

21. Yang M, Wu M, Xia P, Wang C, Yan P, et al. (2012) The role of microtubule-associated protein $1 \mathrm{~B}$ in axonal growth and neuronal migration in the central nervous system. Neural Regen Res 7: 842-8.

22. Yoshimura T, Rasband MN (2014) Axon initial segments: diverse and dynamic neuronal compartments. Curr Opin Neurobiol 27: 96-102.

23. Inagaki N, Chihara K, Arimura N, Menager C, Kawano Y, et al. (2001) CRMP2 induces axons in cultured hippocampal neurons. Nature Neurosci. 4: 781-782.

24. Kawano Y, Yoshimura T, Tsuboi D, Kawabata S, Kaneko-Kawano T, et al. (2005) CRMP-2 is involved in kinesin-1-dependent transport of the Sra-1/WAVE1 complex and axon formation. Mol Cell Biol 25: 9920-9935.

25. Nishimura T, Fukata Y, Kato K, Yamaguchi T, Matsuura Y, et al. (2003) CRMP-2 regulates polarized Numb-mediated endocytosis for axon growth. Nat Cell Biol 5: 819-826.

26. Yoshimura T, Kawano Y, Arimura N, Kawabata S, Kikuchi A, et al. (2005) GSK-3beta regulates phosphorylation of CRMP-2 and neuronal polarity. Cell 120: 137-149.

27. Alabed YZ, Pool M, Ong Tone S, Fournier AE (2007) Identification of CRMP4 as a convergent regulator of axon outgrowth inhibition. J Neurosci 27: 1702-1711.

28. Quach TT, Duchemin AM, Rogemond V, Aguera M, Honnorat J, et al. (2004) Involvement of collapsin response mediator proteins in the neurite extension induced by neurotrophins in dorsal root ganglion neurons. Mol Cell Neurosci 25: 433-443.

29. Bamburg JR, Bloom GS (2009) Cytoskeletal pathologies of Alzheimer disease. Cell Motil Cytoskeleton 66: 635-649.

30. Frisoni GB, Sabattoli F, Lee AD, Dutton RA, Toga AW, et al. (2006) In vivo neuropathology of the hippocampal formation in $\mathrm{AD}$ : a radial mapping MR-based study. Neuroimage 32: 104-110.

31. Williamson R, van Alten L, Mann DM, Platt B, Plattner F, et al. (2011) CRMP2 hyperphosphorylation is characteristic of Alzheimer's disease and not a feature common to other neurodegenerative diseases. J. Alzheimers Dis 27: 615-25.

32. Mucke L, Selkoe DJ (2012) Neurotoxicity of amyloid $\hat{\mathrm{I}}^{2}$-protein: synaptic and network dysfunction. Cold Spring Harb Perspect Med 2: a006338.

33. Cole AR, Noble W, van Aalten L, Plattner F, Meimaridou R, et al. (2007) Collapsin response mediator protein-2 hyperphosphorylation is an early event in Alzheimer's disease progression. J Neurochem 103: 1132-1144.

34. Stauber J, Lemaire R, Franck J, Bonnel D, Croix D, et al. (2008) MALDI imaging of formalin-fixed paraffin-embedded tissues: application to model animals of Parkinson disease for biomarker hunting. J Proteome Res 7: 969-78.

35. Prusiner SB (1998) Prions. Proc Natl Acad Sci U S A 95: 13363-13383.

36. Auvergnon N, Reibel S, Touret M, Honnorat J, Baron T, et al. (2009) Altered expression of CRMPs in the brain of bovine spongiform encephalopathy-infected mice during disease progression. Brain Res 1261: 1-6.

37. Ishikura N, Clever JL, Bouzamondo-Bernstein E, Samayoa E, Prusiner SB, et al. (2005) Notch-1 activation and dendritic atrophy in prion disease. Proc Natl Acad Sci U S A 102: 886-891.

38. Shinkai-Ouchi F, Yamakawa Y, Hara H, Tobiume M, Nishijima M, et al. (2010) Identification and structural analysis of C-terminally truncated collapsin response mediator protein-2 in a murine model of prion diseases. Proteome Sci 8: 53 .
39. Moloney EB, de Winter F, Verhaagen J (2014) ALS as a distal axonopathy: molecular mechanisms affecting neuromuscular junction stability in the presymptomatic stages of the disease. Front Neurosci 14: 8:252.

40. Dadon-Nachum M, Melamed E, Offen D (2011) The "dying-back" phenomenon of motor neurons in ALS. J Mol Neurosci 43: 470-477.

41. Robberecht W, Philips T (2013) The changing scene of amyotrophic lateral sclerosis. Nat Rev Neurosci 14: 248-264.

42. Letournel F, Bocquet A, Dubas F, Barthelaix A, Eyer J (2003) Stable tubule only polypeptides (STOP) proteins co-aggregate with spheroid neurofilaments in amyotrophic lateral sclerosis. J Neuropathol Exp Neurol 62: 1211-1219.

43. Møller RS, Dahl HA, Helbig (2015) The contribution of next generation sequencing to epilepsy genetics. Expert Rev Mol Diagn 15: 1531-1538.

44. Mériaux C, Franck J, Park DB, Quanico J, Kim YH, et al. (2014) Human temporal lobe epilepsy analyses by tissue proteomics. Hippocampus 24: 628-642.

45. Wilson SM, Xiong W, Wang Y, Ping X, Head JD, et al. (2012) Prevention of posttraumatic axon sprouting by blocking collapsin response mediator protein 2-mediated neurite outgrowth and tubulin polymerization. Neuroscience 17: 210:451-466.

46. Dudek FE, Sutula TP (2007) Epileptogenesis in the dentate gyrus: a critical perspective. Prog Brain Res 163: 755-773.

47. Didelot A, Honnorat J (2014) Paraneoplastic disorders of the central and peripheral nervous systems. Handb Clin Neurol 121: 1159-1179.

48. Honnorat (2009) Auto-antibodies against CRMP5 (also called anti-CV2 or anti-CV2/CRMP5) have been described in a subgroup of PNS. J. Neurol Neurosurg Psychiatry 80:412-6.

49. Camdessanché JP, Ferraud K, Boutahar N, Lassablière F, Mutter M, et al. (2012) The collapsin response mediator protein 5 onconeural protein is expressed in Schwann cells under axonal signals and regulates axonSchwann cell interactions. J Neuropathol Exp Neurol 71: 298-311.

50. McGee A, Li G, Lu Z, Qiu S (2014) Convergent synaptic and circuit substrates underlying autism genetic risks. Front Biol (Beijing) 9: 137-150.

51. Chen J, Yu S, Fu Y, Li X (2014) Synaptic proteins and receptors defects in autism spectrum disorders. Front Cell Neurosci 8: 276.

52. Hallmayer J, Cleveland S, Torres A, Phillips J, Cohen B, et al. (2011) Genetic heritability and shared environmental factors among twin pairs with autism. Arch Gen Psychiatry 68: 1095-1102.

53. Braunschweig D, Krakowiak P, Duncanson P, Boyce R, Hansen RL, et al. (2013). Autism-specific maternal autoantibodies recognize critical proteins in developing brain. Transl Psychiatry 3: e77.

54. Ripsch MS, Ballard CJ, Khanna M, Hurley JH, White FA, et al. (2012) A peptide uncoupling CRMP2 from the presynaptic $\mathrm{Ca}(2+)$ channel complex demonstrates efficacy in animal models of migraine and AIDS therapy-induced neuropathy. Transl Neurosci 3: 1-8.

55. Winquist RJ, Pan JQ, Gribkoff VK (2005) Use-dependent blockade of Cav2.2 voltage-gated calcium channels for neuropathic pain. Biochem Pharmacol 70: 489-499.

56. Brittain JM, Duarte DB, Wilson SM, Zhu W, Ballard C, et al. (2011) Suppression of inflammatory and neuropathic pain by uncoupling CRMP-2 from the presynaptic Ca2+ channel complex. Nat. Med 5;17: 822-829.

57. Piekarz AD, Due MR, Khanna M, Wang B, Ripsch MS, et al. (2012) CRMP2 peptide mediated decrease of high and low voltage-activated $\mathrm{Ca} 2+$ channels, attenuation of nociceptor excitability, and anti-noception in a model of AIDS therapy-induced painful peripheral neuropathy. Mol. Pain 8: 1-19.

58. Calon F, Lim GP, Yang F, Morihara T, Teter B, et al. (2004) Docosahexaenoic acid protects from dendritic pathology in an Alzheimer's disease mouse model. Neuron 43: 633-645.

59. Wu F, Catano M, Echeverry R, Torre E, Haile WB, et al. (2014) Urokinase-type plasminogen activator promotes dendritic spine recovery and improves neurological outcome following ischemic stroke. J Neurosci 22;34: 14219-32. 
Citation: Quach TT, Auvergnon N, Lerch JK, Honnorat J, Khanna R, et al. (2016) Cytoskeleton and CRMPs in Neuronal Morphogenesis and Neurological Diseases: Potential Targets for New Therapies. J Neurol Disord 4: 257. doi:10.4172/2329-6895.1000257

Page 8 of 8

60. Kevenaar JT, Hoogenraad CC (2015) The axonal cytoskeleton: from organization to function. Front Mol Neurosci 8: 44.

61. Brunden KR, Trojanowski JQ, Smith AB, Lee VM, Ballatore C (2014) Microtubule-stabilizing agents as potential therapeutics for neurodegenerative disease. Bioorg Med Chem 22: 5040-5049.

62. Dumont RJ, Verma S, Okonkwo DO, Hurlbert RJ, Boulos PT, et al. (2001) Acute spinal cord injury, part II: contemporary pharmacotherapy. Clin. Neuropharmacol 24: 265-79.

63. Zhang B, Carroll J, Trojanowski JQ, Yao Y, Iba M, et al. (2012) The microtubule-stabilizing agent, epothilone $\mathrm{D}$, reduces axonal dysfunction, neurotoxicity, cognitive deficits, and Alzheimer-like pathology in an interventional study with aged tau transgenic mice. J Neurosci 32: 3601-3611.

64. Ballatore C, Brunden KR, Huryn DM, Trojanowski JQ, Lee VM, et al. (2012) Microtubule stabilizing agents as potential treatment for Alzheimer's disease and related neurodegenerative tauopathies. J Med Chem 55: 8979-8996.

65. Ju W, Li Q, Allette YM, Ripsch MS, White FA, et al. (2013) Suppression of pain-related behavior in two distinct rodent models of peripheral neuropathy by a homopolyarginine-conjugated CRMP2 peptide. J Neurochem 124: 869-879.

66. Fischer G, Pan B, Vilceanu D, Hogan QH, Yu H (2014) Sustained relief of neuropathic pain by AAV-targeted expression of $\mathrm{CBD} 3$ peptide in rat dorsal root ganglion. Gene Ther 21: 44-51.

67. Feldman P, Khanna R (2013) Challenging the catechism of therapeutics for chronic neuropathic pain: Targeting CaV2.2 interactions with CRMP2 peptides. Neurosci Lett 557 Pt A: 27-36.

68. Kaneko M, Sugiyama N, Sasayama D, Yamaoka K, Miyakawa T, et al. (2008) Prion disease causes less severe lesions in human hippocampus than other parts of brain. Psychiatry Clin Neurosci 62: 264-270.
69. Quach TT, Wang Y, Khanna R, Chounlamountri N, Auvergnon N, et al. (2011) Effect of CRMP3 expression on dystrophic dendrites of hippocampal neurons. Mol Psychiatry 16: 689-691.

70. Ip JP, Fu AK, Ip NY (2014) CRMP2: functional roles in neural development and therapeutic potential in neurological diseases. Neuroscientist 20: 589-598.

71. Connolly S, Galvin M, Hardiman O (2015) End-of-life management in patients with amyotrophic lateral sclerosis. Lancet Neurol 14: 435-442.

72. Duplan L, Bernard N, Casseron W, Dudley K, Thouvenot E, et al. (2010) Collapsin response mediator protein $4 \mathrm{a}$ (CRMP4a) is upregulated in motoneurons of mutant SOD1 mice and can trigger motoneuron axonal degeneration and cell death. J Neurosci 30: 785-796.

73. Moutal A, François-Moutal L, Perez-Miller S, Cottier K, Chew LA, et al. (2015) (S)-Lacosamide Binding to Collapsin Response Mediator Protein 2 (CRMP2) Regulates CaV2.2 Activity by Subverting Its Phosphorylation by Cdk5. Mol Neurobiol.

74. François-Moutal L, Wang Y, Moutal A, Cottier KE, Melemedjian OK, et al. (2015) A membrane-delimited N-myristoylated CRMP2 peptide aptamer inhibits CaV2.2 trafficking and reverses inflammatory and postoperative pain behaviors. Pain 156: 1247-1264.

75. Yamashita N, Goshima Y (2012) Collapsin response mediator proteins regulate neuronal development and plasticity by switching their phosphorylation status. Mol Neurobiol 45: 234-246.

76. Coles CH, Bradke F (2015) Coordinating neuronal actin-microtubule dynamics. Curr Bio 25: R677-691. 